\title{
Downscaling Gridded DEM using the Hopfield Neural Network
}

\author{
Nguyen Quang Minh, Nguyen Thi Thu Huong, La Phu Hien, H.G. Lewis, P.M. Atkinson
}

\begin{abstract}
A new Hopfield neural network (HNN) model for downscaling a digital elevation model in grid form (gridded DEM) is proposed. The HNN downscaling model works by minimizing the local semivariance as a goal, and by matching the original coarse spatial resolution elevation value as a constraint. The HNN model is defined such that each pixel of the original coarse DEM is divided into $f \times f$ sub-pixels, represented as network neurons. The elevation of each sub-pixel is then derived iteratively (i.e. optimized) based on minimizing the local semivariance under the coarse elevation constraint. The proposed HNN model was tested against three commonly applied alternative benchmark methods (bilinear resampling, bi-cubic and Kriging resampling methods) via an experiment using both degraded and sampled datasets at 20 $\mathrm{m}, 60 \mathrm{~m}$ and $90 \mathrm{~m}$ spatial resolutions. For this task, a simple linear activation function was used in the HNN model. Evaluation of the proposed model was accomplished comprehensively with visual and quantitative assessment against the benchmarks. Visual assessment was based on direct comparison of the same topographic features in different downscaled images, scatterplots and DEM profiles. Quantitative assessment was based on commonly used parameters for DEM accuracy assessment such as the root mean square error (RMSE), linear regression parameters $m$ and $b$, and the correlation coefficient $R$. Both visual and quantitative assessment revealed the much greater accuracy of the HNN model for increasing the grid density of gridded DEMs.
\end{abstract}

Index Terms-Digital Elevation Model, Downscaling, Hopfield Neural Networks.

\section{INTRODUCTION}

$\mathrm{T}$ HE spatial resolution of a gridded DEM affects both the information content and the accuracy of the data and, potentially, of many other secondary data products [1], [2]. Examples include the well-known effects of spatial resolution on DEM-related spatial properties [3], [4] such as slope and aspect [5], [6], watershed boundary delineations and soil-wateratmosphere transfer schemes [7], [8], water run-off models [9], [10], three-dimensional modelling of landscapes [11], local slope, plan curvature, drainage area [12], [13], soil survey variables and soil moisture [14], [15]. All of these studies

Manuscript received September 24, 2018. This work was supported by Vietnam National Foundation of Science and Technology Development under the project 105.99-2014.25.

Nguyen Quang Minh (corresponding author) and Nguyen Thi Thu HUONG are with the Faculty of Geomatics and Land Administration, Hanoi University of Mining and Geology, Hanoi, Vietnam (e-mail: nguyenquangminh@humg.edu.vn; nguyenthithuhuong@humg.edu.vn;).

La Phu HIEN is currently with the Department of Geomatics and Surveying, Thuyloi University, Vietnam (email: laphuhien@tlu.edu.vn) showed that DEMs with a finer spatial resolution can produce more informative and potentially more accurate results.

Gridded DEMs with fine spatial resolution and high accuracy can be acquired using remote sensing and airborne LiDAR technology, ground surveying or photogrammetry [16], [17]. Airborne LiDAR enables the acquisition of data with a very high density of 3-dimensional coordinate points and, therefore, production of a DEM with sub-meter spatial resolution. Airborne LiDAR-derived DEMs have been used in many different applications, some of which require very fine spatial resolution and very high accuracy [18]. Although being capable of generating a fine spatial resolution DEM, airborne LiDAR technology has some challenges such as the very large amount of data storage and extensive computing resource required for data processing. Compared with airborne LiDAR, other methods for fine spatial resolution DEM acquisition such as ground surveying and photogrammetry are more time consuming and labour intensive [19]. Hence, if the spatial resolution of the DEM can be increased using algorithms, it is possible to save time and labour costs.

Sometimes, it is necessary to resample a coarse resolution raster DEM to create a DEM with a finer grid spacing and a common approach is to use algorithms such as nearest neighbor, bilinear and bi-cubic interpolation. That is, these algorithms are used to interpolate the raster DEM data [20] such that the resolution or the accuracy of the raster DEM is increased slightly [21], [22]. Another method for resampling gridded DEM data to create a finer grid with higher accuracy is Kriging interpolation [23]. Dixon and Earls [24] used simple nearest neighbor resampling to increase the grid density of DEMs and compare the effects of this for DEM products such as stream flow, watershed, delineations, number of sub-basins and slopes. It was clear that simple nearest neighbor DEM resampling did not increase the accuracy of the DEM greatly, or the resampling methods did not create new significant information that is not available at the original resolution of the DEM [25]. The experiments by Rees [26] and Shi et. al. [22] showed that bilinear, bicubic and Kriging resampling increased

H. Lewis is with the Astronautics Research Group, University of Southampton, Highfield, Southampton SO17 1BJ, UK (e-mail: hglewis@soton.ac.uk)

P. M..Atkinson, was with Geography and Environment, University of Southampton, Highfield, Southampton SO17 1BJ, UK. He is now with the Faculty of Science and Technology, Engineering Building, Lancaster University, Lancaster LA1 4YR. He also is visiting professor at the School of Geography, Archaeology and Palaeoecology, Queen's University Belfast, BT7 1NN, Northern Ireland, UK (e-mail: pma@lancaster.ac.uk). 
the accuracy of DEMs in terms of the root mean square error (RMSE) given a suitable value of re-sampling ratio $r$. Comparing three resampling methods, Kriging performed more accurately than the other two methods for smooth terrain. However, for rough terrain, the accuracies of the three resampling methods were similar [26]. Nevertheless, all studies suggested that the resampling of raster DEM data can potentially increase the resolution and accuracy of the data.

The accuracy of a gridded DEM can be increased by combining the original DEM with additional elevation data. Jana et. al. [27] and Jordan [28] increased the accuracy of a DEM from raster contour and ridgeline elevation data. These methods used additional information from raster channels to correct the elevation values in the DEM cells. Similarly, the accuracy and details of a gridded DEM can be increased using geostatistical methods and sets of additional high accuracy elevation data points [29], [30]. Other methods include B-spline resampling and the filtering method used in a patent by Atkins et al. [31]. This research showed that integrating additional elevation data through algorithms can increase the detail and accuracy of a DEM. The focus of this paper, in contrast, is to increase a DEM's detail and accuracy through downscaling when new measured data at the sub-pixel level are not available.

Sub-pixel mapping is a technique used in remote sensing to predict land cover class at the sub-pixel scale (i.e., at a spatial resolution that is finer than the original input data) using a softclassified land cover proportions image as input [32]. In terms of geographical scaling, sub-pixel mapping approaches are downscaling techniques which use the soft-classified land cover proportions as a pixel-level constraint and maximize some goal functions (e.g., the spatial dependence between sub-pixels) to increase the spatial resolution of the land cover classification [33]. Several sub-pixel mapping techniques have been developed such as the sub-pixel swapping [34], [35], Markov random field [36], geospatial and Hopfield neural network (HNN) approaches [37], [38], [39], [40], [41]. The HNN technique has been modified previously for smoothing and increasing the spatial resolution of raw multispectral remotely sensed imagery [42]. Because remote sensing images and gridded DEMs are both provided in the raster data model, it may be expected that the HNN approach developed for remote sensing images may, with appropriate adjustment, have some utility for increasing the detail and accuracy of gridded DEMs. This paper, therefore, explores the potential for development and application of a new HNN model for downscaling DEM imagery. Specifically, a new and simple HNN was developed for resampling a coarse resolution gridded DEM to create a finer gridded DEM (i.e., with a denser grid) that is closer to the real Earth's surface than both the original DEM and finer grid DEMs generated by several benchmark methods (common resampling approaches such as bilinear, bi-cubic and Kriging interpolation). The proposed HNN model uses the spatial dependence maximization and coarse elevation constraint functions to produce a DEM on a finer grid and with greater accuracy than that of the original DEM. The advantage of the HNN model is, thus, drawn from knowledge of the real world; specifically that the elevation of a sub-pixel is likely to be more similar to the elevations of its adjacent sub-pixels than subpixels that are further away (Tobler's first law of geography). This information is novel relative to the coarse DEM (whereas the coarse elevation constraint is not) and explains the advantage imparted by application of the HNN downscaling operation.

\section{MethoD}

\section{A. HNN approach for sub-pixel mapping}

The model proposed here for increasing the grid density of a gridded DEM is based on the HNN designed for sub-pixel mapping in remote sensing [37], [43]. In the HNN for sub-pixel mapping, an original pixel is divided into $f \times f$ sub-pixels and each sub-pixel is represented by a neuron in the HNN. This particular model is based on an area proportion constraint and two goal functions. The proportion constraint ensures that the total number of sub-pixels of each land cover class in each pixel is equal to the number of sub-pixels that would be assigned by the soft-classified land cover proportion in each coarse pixel. The goal functions play the role of a spatial dependence engine, which increases the tendency of adjacent sub-pixels to belong to the same land cover class.

In the HNN used for sub-pixel mapping, the output $v_{\mathrm{ij}}$ of a neuron (sub-pixel) $(i, j)$ is:

$$
v_{i j}=g\left(u_{i j}\right)=\frac{1}{2\left(1+\tanh \lambda u_{i j}\right)}
$$

here $g\left(u_{i j}\right)$ is an activation function of each neuron, $u_{i j}$ is the input value of each neuron and $\lambda$ is steepness value, which is defined empirically as 100 .

The input value $u_{i j}$ is determined at the time $t$ as

$$
u_{i j}(t)=u_{i j}(t-d t)+\frac{d u_{i j}}{d t} d t
$$

where, $d t$ is time step, $u_{i j}(t-d t)$ is the output value at the time $(t-d t)$ and $d u_{i j} / d t$ is defined as follows:

$$
\frac{d u_{i j}}{d t}=\frac{d E_{i j}}{d v}
$$

where, $E$ is the energy, defined as $E=$ Goals + Constraint and

$$
\frac{d E_{i j}}{d v}=\left(\sum_{e}^{K} \frac{d G_{o a l}}{d v}+\frac{d \text { Constraint }}{d v}\right)
$$

where, $K$ is the number of Goal functions. Depending on the specific application, the goal and constraint functions can be modified for optimization. In Tatem et al. [1], the Goal functions are the two Goal functions for spatial dependence maximization, and the Constraint Functions comprise an Area Constraint function used for retaining the area proportions predicted by the soft-classification and a Multi-class Function which ensures that a sub-pixel belongs to only one class. In Nguyen et. al. [43] a Panchromatic Constraint Function was added to the HNN model of Tatem et. al. [37] to increase the accuracy of the sub-pixel mapping results.

The running of the HNN in the above cases is terminated when the total energy $E$ of the HNN reaches a minimum value determined as

$$
\begin{aligned}
& E=\sum_{i} \sum_{j}\left(\sum_{f}\left(k_{f} v_{i j}^{\text {Goal }_{f}}\right)+\sum_{g}\left(k_{g} v_{i j}^{\text {Constraint }_{g}}\right)\right)=\min \\
& \text { Or: } \quad E(t)-E(t-d t)=0
\end{aligned}
$$




\section{B. Proposed HNN approach for gridded DEM downscaling}

The newly proposed approach is based on the expectation that the elevation of each sub-pixel is likely to be more similar to its adjacent sub-pixels than to more distant sub-pixels (spatial dependence assumption). The realization of spatial dependence in this case is calculated using the semivariance, which can be defined as

$$
\gamma(h)=\frac{1}{2 N(h)} \sum_{1}^{N(h)}\left[v_{i j}-v_{i j+}\right]^{2}
$$

where, $\gamma(h)$ is the semivariance value at lag distance $h$ (i.e., ignoring direction), $h$ is the distance between a pair of data points $v_{i j}$ and $v_{i j+h}$, and $N(h)$ is the number of pairs of data points. If the points are spatially dependent, the semivariance will be small at small value of $h$. In other words, there is greater spatial dependence when there is a large difference between the a priori variance (maximum fitted semivariance at large $h$ ) and the smallest semivariance at small $h$. Thus, minimizing the semivariance at small $h$ (at the sub-pixel scale) effectively maximizes the spatial dependence, that is, creates the greatest amount of spatial structure in the DEM at fine spatial resolution. The minimum value of semivariance can be defined based on the derivative as

$$
\frac{\partial \gamma(h)}{\partial v}=0
$$

and,

$\frac{\partial \gamma(h)}{\partial v}=\frac{1}{2 N(h)} \sum_{1}^{N(h)}\left(2 v_{i j}-2 v_{i j+h}\right)=v_{i j}-\frac{\sum_{1}^{N(h)} v_{i j+h}}{N(h)}$

So, from Equation (7), it is possible to achieve an expected output value of

$$
v_{i j}^{\text {expected }}=\frac{\sum_{1}^{N(h)} v_{i j+h}}{N(h)}
$$

The change in elevation of each sub-pixel from the spatial dependence maximization operation is

$$
d u_{i j}^{s d}=v_{i j}^{\text {expected }}-v_{i j}
$$

This means that the expected value $v_{i j}$ is the average of the values of all data points at lag distance $h\left(v_{i j+h}\right)$. In this model, for a grided DEM, the data points with smallest $h$ are the eight sub-pixels surounding the sub-pixel. We term this function the spatial dependence maximization function. Similar to two Goal functions in the HNN model proposed by Tatem et al. [37] the spatial dependence maximization function also maximizes the spatial dependence between the adjacent sub-pixels. However, the difference between these functions is that while the Goal functions of Tatem et al. increase the value of the central subpixel to 1 or reduce the value of the central sub-pixel to 0 , the spatial dependence maximization function in the new HNN model increases or reduces the output value of the central subpixel to the average elevation of the eight surrounding subpixels.

The proposed model developed for downscaling a gridded DEM is presented in Fig. 1 for the example of a coarse DEM with $2 \times 2$ pixels. A pixel in the original DEM is divided into $4 \times 4$ sub-pixels in the new DEM (zoom factor $f=4$ ). So the original image of $2 \times 2$ pixels is resampled to an image of $8 \times 8$ sub-pixels. Each sub-pixel is represented by a neuron in the HNN model where the initial value is the elevation value of the pixel in the original DEM (or may be assigned randomly). According to

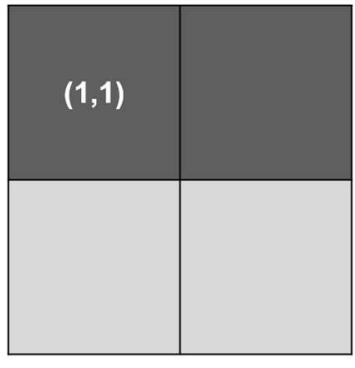

Original DEM

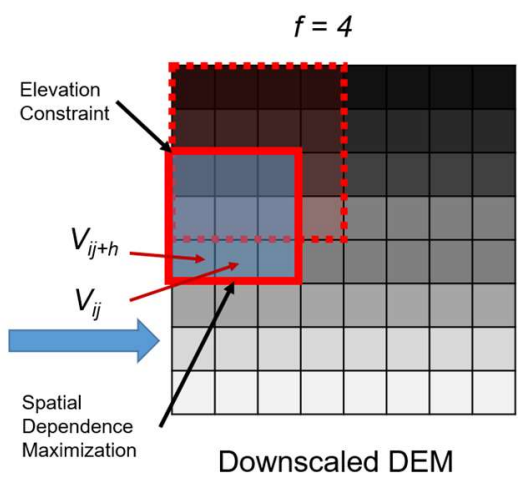

Downscaled DEM
Fig. 1 Downscaling of grid DEM by a factor of 4

Equation (10), the expected elevation for each sub-pixel is equal to the average of the eight surounding sub-pixels (based on the spatial dependence maximization function using a $3 \times 3$ window).

If the spatial dependence maximizing function is the only function used in the model, the elevation of all sub-pixels in the new DEM will be finally the same and the elevation values of the coarse original DEM will not be preserved. To resolve this problem, a simple constraint function is used. The aim of this constraint is to achieve theoretically perfect consistency between scales; the elevation of a pixel of the coarse DEM represents the average elevations of all sub-pixels within that pixel. That is, the average elevation of all sub-pixels located within a pixel of the original DEM must be equal to the elevation of that original pixel. For example, the average of the elevation of all sub-pixels within the area of the pixel $(1,1)$ of the original image in Fig. 1 must be equal to the elevation of the pixel $(1,1)$.

$d u_{i j}^{e p}=$ Elevation $_{x, y}-\frac{\sum_{(x-1) f}^{x f} \sum_{(y-1) f}^{y f} v_{p q}}{f \times f}$

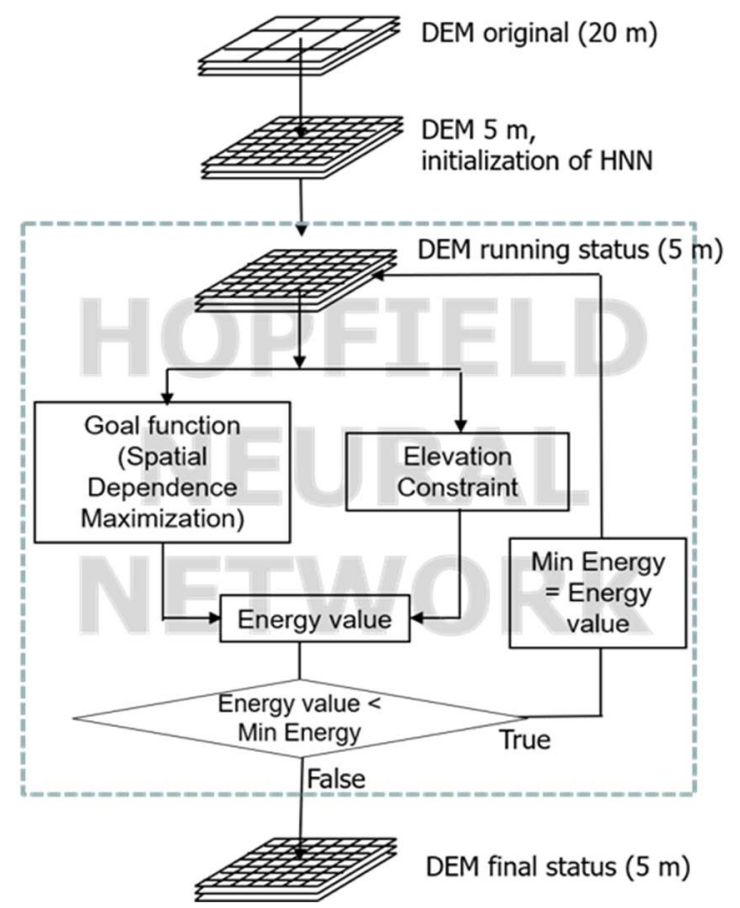

Fig. 2 Hopfield Neural Network Model for DEM downscaling (illustrating the example of downscaling from $20 \mathrm{~m}$ to $5 \mathrm{~m}$ ) 
where Elevation $n_{x, y}$ is the elevation value of pixel $(x, y)$ in the original image, $v_{p q}$ is the output (elevation) value of the subpixel $(p, q)$ in the newly generated image covered by pixel $(x$, $y$ ), and $f$ is the zoom factor. If the average of the elevation values of all sub-pixels within a pixel is smaller than the Elevation $_{x, y}$, then the elevation values $v_{p q}$ of all sub-pixels within the footprint of pixel $(x, y)$ are increased. In contrast, when the average of the elevation values of all sub-pixels within pixel $(x, y)$ is larger than the Elevation ${ }_{x, y}$, a value is subtracted from the output value $v_{p q}$ of the neuron $(p, q)$.

Given the above, the input value of each neuron (sub-pixel) can be calculated based on Equation (2) with the value $d u_{i j} / d t$ as

$$
\frac{d u_{i j}}{d t}=\frac{d E_{i j}}{d v}=d u_{i j}^{s d}+d u_{i j}^{e p}
$$

The output value $v_{i j}$ of each neuron is then calculated using an activation function $g\left(u_{i j}\right)$. However, in this new HNN model, the activation function $g\left(u_{i j}\right)$ is not the same as in Equation 1 because it is not used to push the output value of the neurons to 0 or 1 as for sub-pixel mapping. Instead, a linear activation function, as presented in Tank and Hopfield [44], was used in this new approach as

$$
v_{i j}=g\left(u_{i j}\right)=a u_{i j}+b
$$

where, $a=1$ and $b=0$ in this model.

The HNN network runs until the energy $E$ value is minimized as

$$
E=\sum_{i} \sum_{j}\left(d u_{i j}^{s d}+d u_{i j}^{e p}\right)=\min
$$

or, the $E(t)-E(t-d t)=0$, where $(t-d t)$ and $t$ are two consecutive iterations of the HNN.

Another difference between the HNN model for sub-pixel mapping and the HNN model for DEM downscaling is in the constants $K$ in Equation (5). These $K$ values were used to control the effect of the goal and constraint functions. Because the activation function of the proposed HNN for DEM downscaling is a linear function, $K$ no longer provides a useful effect.

Fig. 2 shows a flowchart of the modified HNN model for DEM downscaling illustrating the example of downscaling from an original $20 \mathrm{~m}$ to a fine $5 \mathrm{~m}$ resolution. Firstly, the $20 \mathrm{~m}$ original DEM is resampled to $5 \mathrm{~m}$ using a simple resampling method such as nearest neighbor to provide the initial status of the HNN. Each $5 \mathrm{~m}$ sub-pixel represented by a neuron in the $\mathrm{HNN}$ and the initial input values of the neurons are the elevation values of the sub-pixels. The output values of the neurons of the $\mathrm{HNN}$ (or the elevation values) are calculated based on the goal function and elevation constraint. The sum of all goal function (Equation 10) and elevation constraint (Equation 11) values is the energy function value (Equation 14). If the total energy function value of the network is not minimized, then the Min Value is new energy value and the HNN will run for a further iteration. The iterative process continues until the energy function value of two consecutive iterations does not change and the final output value of each neuron in the HNN is the elevation of the sub-pixel corresponding to this neuron.

\section{ASSESSMENT OF THE ALGORITHM}

\section{A. Reference and testing data}

Two types of data were used to evaluate the proposed HNN model. The first type of data consisted of degraded coarse DEMs which were calculated from reference DEMs at fine resolution using simple averaging to create the source data for algorithm testing. Elevation values of pixels in these coarse degraded DEM datasets do not contain interpolation and measurement errors. These elevation values contain only very small or negligible calculation errors. The difference between the Earth's surface depicted by these degraded DEMs and that depicted by the reference DEMs is due only to the difference in pixel size. So, these data are helpful for method evaluation. These data may be enough to assess the algorithm, but their use alone may lead to some skepticism because they may be thought of as "simulated" and are not obtained from measured elevation data (e.g., ground measuring, LiDAR) or contour data. Actually, the value in a pixel of a DEM represents the elevation of the surface covered by this pixel, that is, it represents the average elevation of this surface. Interpolation algorithms are used to estimate this elevation from point or contour data such that, again, the elevation of a pixel in a gridded DEM is the average elevation of all possible points within the footprint of each pixel with some estimation errors. Nevertheless, to provide a more comprehensive assessment of the new HNN model, (non-degraded) DEM data generated by interpolating point and contour data were also used directly for evaluation.

The spatial resolutions of all four testing DEM datasets in this paper were selected to be between $5 \mathrm{~m}$ and $90 \mathrm{~m}$ and the zoom factors were chosen to be 3 or 4 . There are two reasons for this choice of spatial resolution. The first is because most

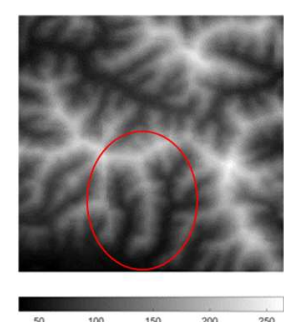

(a)

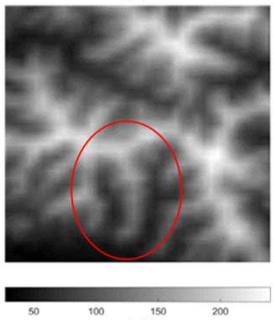

(d)

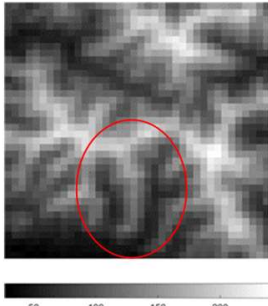

(b)

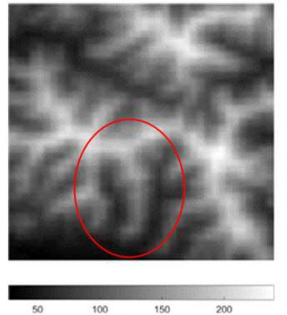

(e)

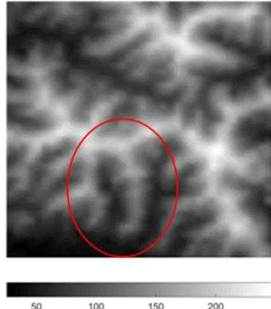

(c)

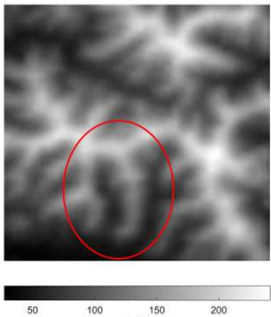

(f)
Fig. 3 Downscaling of DEM from $60 \mathrm{~m}$ to $20 \mathrm{~m}$ spatial resolution. (a) Reference DEM at $20 \mathrm{~m}$ resolution; (b) Input coarse (Degraded) DEM at $60 \mathrm{~m}$ resolution (note: this forms the only input to the algorithms); (c) HNN downscaled DEM at $20 \mathrm{~m}$ resolution; (d) DEM at $20 \mathrm{~m}$ using bilinear resampling; (e) DEM at 20 $\mathrm{m}$ resolution using bi-cubic resampling; (f) DEM at $20 \mathrm{~m}$ resolution using Kriging resampling; 

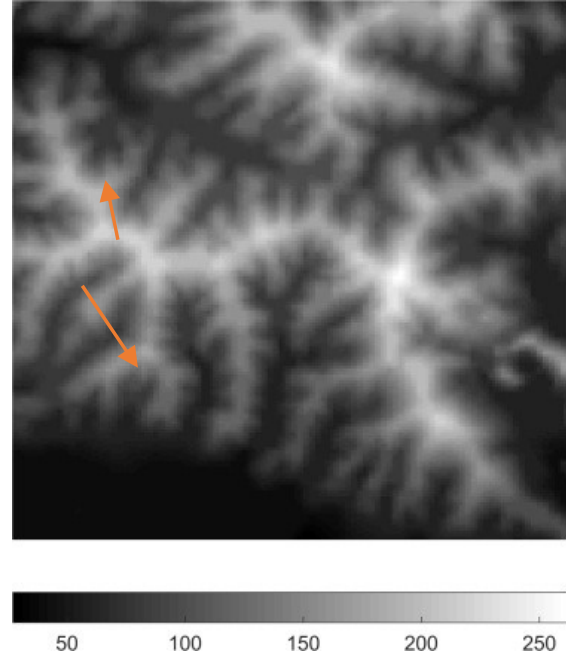

(a)
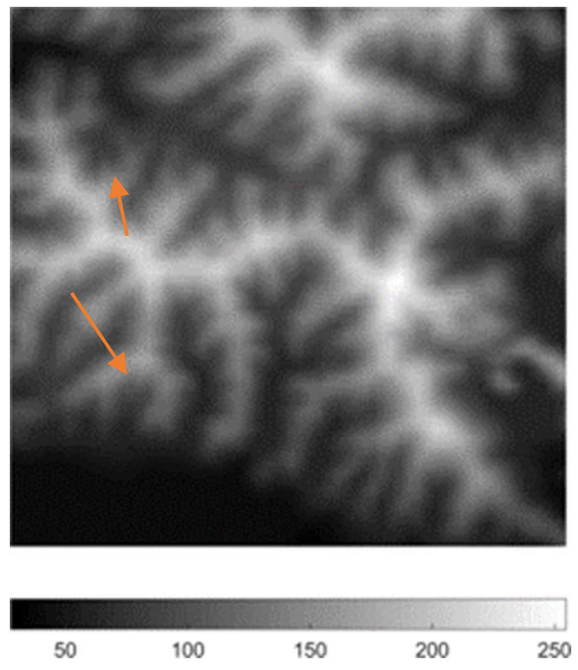

(d)
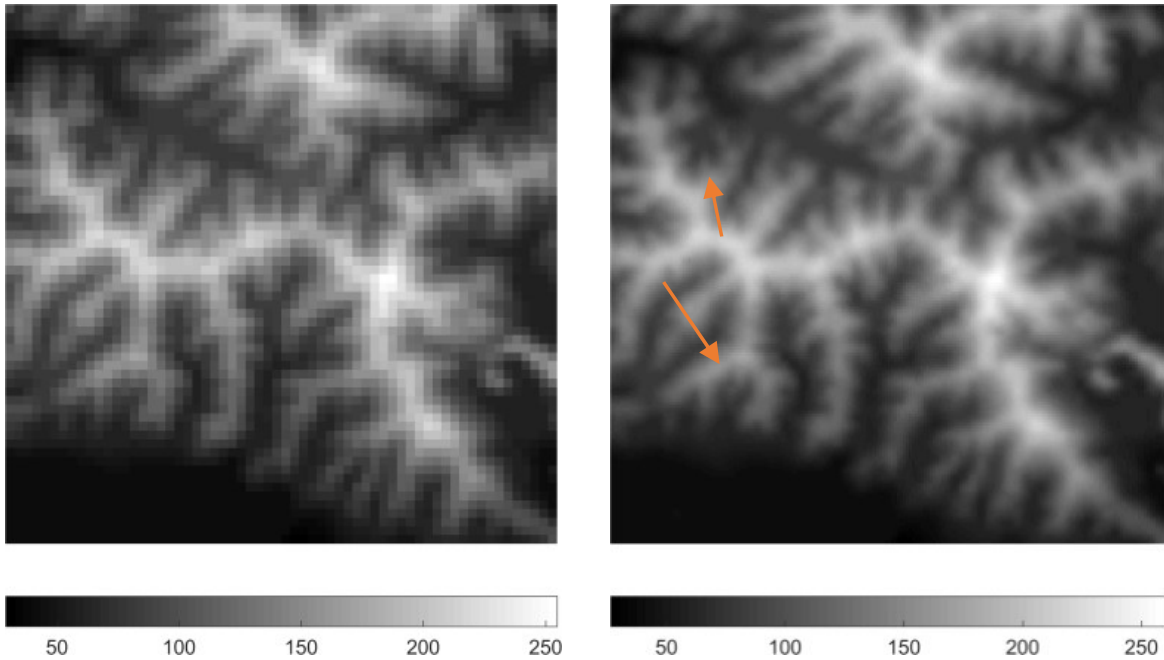

(b)
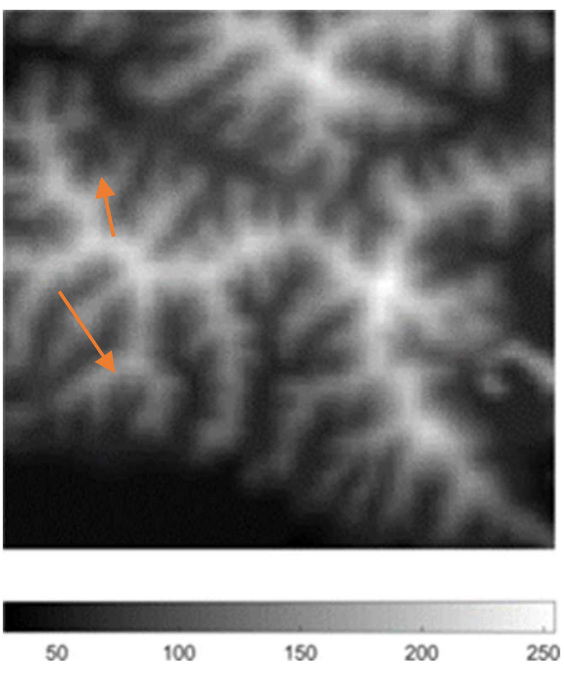

(e)

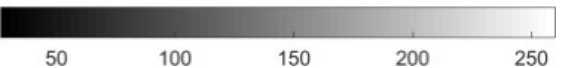

(c)

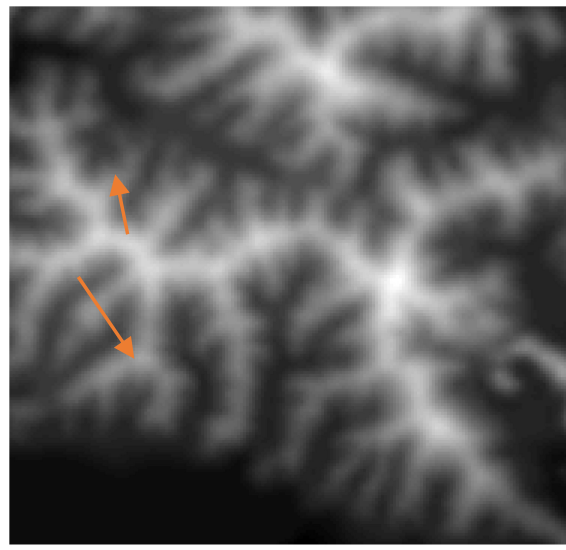

50

100

(f)

Fig. 4 Downscaling of DEM from $90 \mathrm{~m}$ to $30 \mathrm{~m}$ spatial resolution (D2 dataset) (a) Reference DEM at $30 \mathrm{~m}$ resolution; (b) Input coarse (degraded) DEM at $90 \mathrm{~m}$ resolution (note: this forms the only input to the algorithms); (c) HNN downscaled DEM at $30 \mathrm{~m}$ resolution; (d) DEM at $30 \mathrm{~m}$ resolution resulted from bilinear resampling; (e) DEM 30 m resolution resulted from bi-cubic resampling; (f) DEM at 30 m resolution resulted by Kriging resampling;

gridded DEM data currently available are at this range of resolutions. The second and more important reason is that the potential increase in the grid density and, thereby, detail of these commonly available datasets may be useful for a wide range of applications. Fine spatial resolution gridded DEM data may be obtained from airborne LiDAR or 3D Laser scanners, but they are sufficiently resolved for most common applications such that increasing the grid density of these data is less likely to be required.

\section{Degraded DEMs}

The first set of degraded DEM data (termed the D1 dataset) covered an area of about $3.5 \mathrm{~km}$ by $3.5 \mathrm{~km}$ and was acquired at Yen Thanh District, Nghe An Province, in North Central Vietnam. The area is located at $18^{\circ} 58^{\prime} 57.03^{\prime \prime} \mathrm{N}, 105^{\circ} 22^{\prime}$ 44.87" E, about $45 \mathrm{~km}$ from Vinh City. This DEM was produced from topographic maps at a scale of 1:10000. The spatial resolution of the original DEM is $20 \mathrm{~m}$ (Fig. 3(a)) and this was degraded to $60 \mathrm{~m}$ by averaging the elevation values of $20 \mathrm{~m}$ pixels within the footprints of the degraded $60 \mathrm{~m}$ pixels
(Fig. 3(b)).

The second degraded DEM dataset (termed the D2 dataset) was provided by the USGS Earth Explorer acquired by the Shuttle Radar Topography Mission (SRTM) (Fig. 4(a)). This dataset covered the same area as the D1 dataset, but with a spatial resolution of $30 \mathrm{~m}$. This was also degraded to $90 \mathrm{~m}$ to create a second set of test data for the HNN model (Fig. 4(b)).

\section{Sampled DEMs}

The first sampled dataset (termed the S1 dataset) was acquired using ground surveying in Lang Son Province of Vietnam. The area of the test field is about $200 \mathrm{~m}$ by $200 \mathrm{~m}$ in Mai Pha Ward, Lang Son City which is about $150 \mathrm{~km}$ from Hanoi. A set of 533 measured elevation points was used with Kriging interpolation to generate a gridded DEM dataset at $5 \mathrm{~m}$ grid spacing for use as a reference, as can be seen in Fig. 4(a). The accuracy of the reference DEM was assessed with a set of 234 validation points [45], [46]. The results of accuracy assessment for the $\mathrm{S} 1$ dataset (Table 1) showed that the quality of the reference DEM is slightly greater than that of the ASPRS 


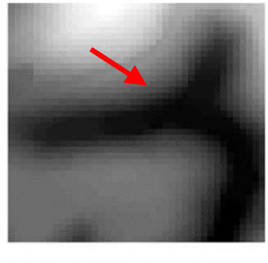

(a)

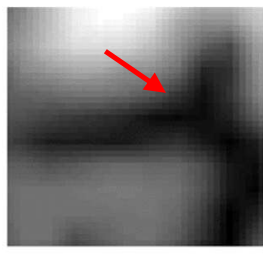

(d)

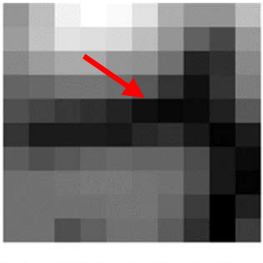

(b)

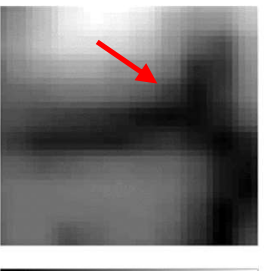

(e)

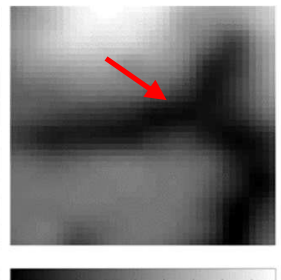

(c)

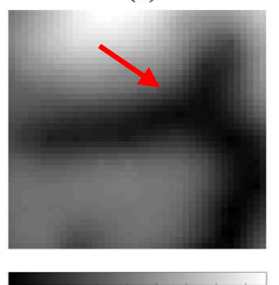

$(f)$
Fig. 5 Downscaling of DEM data for S1 dataset (a) Reference DEM data at 5 $\mathrm{m}$ resolution; (b) Input coarse DEM data at $20 \mathrm{~m}$ resolution (note: this forms the only input to the algorithms); (c) HNN downscaled DEM at $5 \mathrm{~m}$ resolution; (d) DEM at $5 \mathrm{~m}$ resolution resulted from bilinear resampling; (e) DEM at $5 \mathrm{~m}$ resolution resulted from bi-cubic resampling; (f) DEM at $5 \mathrm{~m}$ resolution resulted from Kriging resampling;

TABLE I

ACCURACY ASSESSMENT BASED ON ASPRS ACCURACY STANDARD FOR DigitAL GEOSPATIAL DATA

\begin{tabular}{|c|c|c|c|c|}
\hline \multirow[b]{2}{*}{$\begin{array}{l}\text { Dataset and } \\
\text { standards }\end{array}$} & \multicolumn{3}{|c|}{ Absolute Accuracy } & \multirow[b]{2}{*}{$\begin{array}{c}\text { Appropriate } \\
\text { Contour } \\
\text { Interval } \\
\text { Supported by } \\
\text { the RMSEz } \\
\text { value }\end{array}$} \\
\hline & $\begin{array}{l}\text { RMSE }_{z} \\
\text { Non- } \\
\text { Vegetated } \\
\text { (cm) }\end{array}$ & $\begin{array}{c}\text { NVA at } \\
95 \% \\
\text { Confidence } \\
\text { Level }(\mathrm{cm})\end{array}$ & $\begin{array}{l}\text { VVA at } \\
\text { 95th } \\
\text { Percentile } \\
\text { (cm) }\end{array}$ & \\
\hline $\begin{array}{c}\text { Mai Pha, } \\
\text { Langson DEM }\end{array}$ & 48.3 & 144.9 & 144.9 & 144.9 \\
\hline $\begin{array}{c}\text { Standard } \\
\text { ASPRS class } \\
\text { VIII }(66.7-\mathrm{cm})\end{array}$ & 66.7 & 200 & 200 & 200 \\
\hline
\end{tabular}

Accuracy Standard for Digital Geospatial Data of $66.7 \mathrm{~cm}$ ASPRS DEM Class and Class VIII of ASPRS 1990 Standards [46] with a RMSE of $48.3 \mathrm{~cm}$. The appropriate spatial resolution was created using the same interpolation contour interval of $1.449 \mathrm{~m}$. The coarse DEM at $20 \mathrm{~m}$ spatial resolution was created using the same interpolation algorithm from the point data (Fig. 5(b)). This coarse resolution $20 \mathrm{~m}$ DEM was used as input for the HNN model to downscale to the $5 \mathrm{~m}$ DEM and this result was compared with $5 \mathrm{~m}$ DEM reference data.

The second sampled DEM dataset (termed the S2 dataset) was created from contour data in Dac Ha district in Kontum Province in Vietnam. The location of this DEM dataset was at $14.671794^{\circ} \mathrm{N}$ and $107.967292^{\circ} \mathrm{E}$. The area of the test field is about $6.6 \mathrm{~km}$ by $6.6 \mathrm{~km}$. From the original contour data at $5 \mathrm{~m}$ interval (Fig. 6(a)), a $30 \mathrm{~m}$ resolution DEM was generated and used as reference data (Fig. 6(c)). The coarse $90 \mathrm{~m}$ spatial resolution data (Fig. 6(d)) were then interpolated from the $10 \mathrm{~m}$ interval contours of the same area (Fig. 6(b)). The evaluation was then implemented by comparing the resulting $30 \mathrm{~m}$ DEMs downscaled from the coarse $90 \mathrm{~m}$ DEMs (zoom factor of 3) with the $30 \mathrm{~m}$ reference data.

\section{B. Accuracy Assessment}

\section{1) Results and assessment methods}

To test the proposed HNN model, the DEMs with coarser spatial resolution were used as an input to produce DEMs at the same resolution as the reference data using the bilinear, bi-cubic and Kriging resampling approaches and the HNN downscaling model. In the experiment with Kriging resampling, several Kriging parameters such as semivariogam models, number of observations and the range of searching for observations were tested. The optimal for Kriging resampling were the exponential variogram model with eight observations. A computer program for the HNN downscaling was created using Visual Basic 6 . The HNN downscaling program was performed on a desktop computer with Intel Pentium 5 Processor and $8 \mathrm{~GB}$ RAM. For the $\mathrm{S} 1$ dataset, the running time was 2 seconds after 53 iterations. For the other three test datasets (D1, D2, S2), the running times were from 5 minutes to 7 minutes, depending on the sizes of the DEMs. This running time is more than for the conventional resampling methods, each of which took less than a minute for all four datasets. Results of these downscaling methods for the four datasets are presented in Fig. 3, Fig. 4, Fig. 5 and Fig. 6.

The assessment was implemented based on both visual comparison of the resulting DEMs from the four different methods and quantitative evaluation using the parameters which were usually used for DEM accuracy assessment such as RMSE [47], coefficient of determination [48], linear regression parameters, and the elevation profiles [47], [49].

Visual assessment of the results was carried out by several approaches. The first approach was direct visual comparison of the DEM images, especially comparison of the same topographical features in different images. The second approach was to analyse the scatterplots between the elevation values of the pixels of the reference DEM and the elevation values of the corresponding pixels of the HNN downscaled DEM, and the benchmark DEMs as in Fig. 9 and Fig. 10.

Another approach used in previous research on DEM evaluation involves comparing the cross-sections (profiles) of the resulting downscaled DEMs [45], [46]. These profiles present the match between the surfaces formed by the reference fine grid DEM and the surfaces formed by the DEM at coarse spatial resolution, DEMs generated by the benchmark approaches, and the HNN downscaling model and, thereby, enable evaluation of the effects of the algorithms on different forms of terrain and topographical features. The profiles for the four datasets are presented in Fig. 7.

Quantitative assessment was implemented based mainly on the RMSEs for the whole DEMs and profiles of the four datasets as presented in Table 2, where the overall RMSE, and profiles of the largest and smallest RMSE improvements are shown. Together with the RMSEs, linear regression coefficients such as slope $m$, intercept $b$, and correlation $R$ were used to assess the match between the downscaled DEMs and the reference DEMs for the four datasets (Table 3).

2) Visual assessment

Visual comparison showed that the resulting DEMs from the 


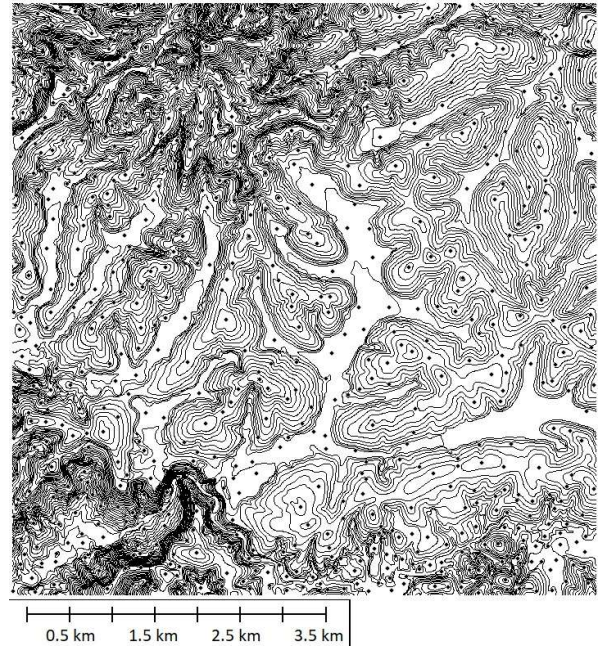

(a)

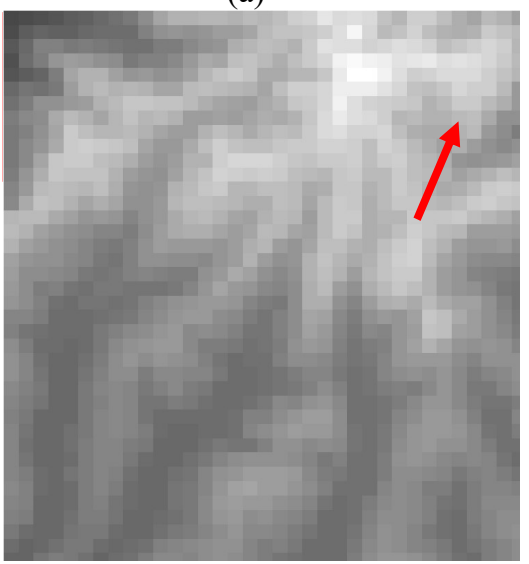

(d)

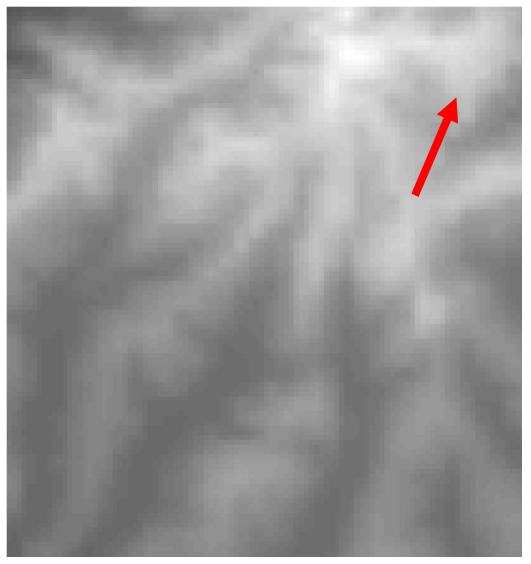

(g)

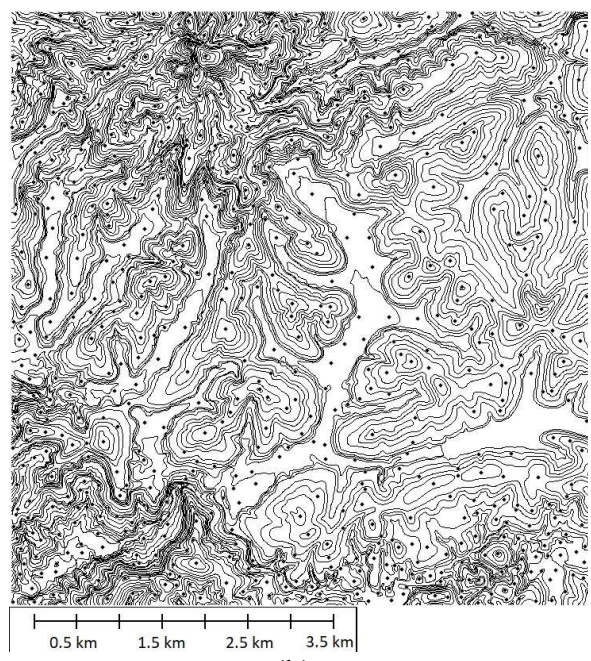

(b)

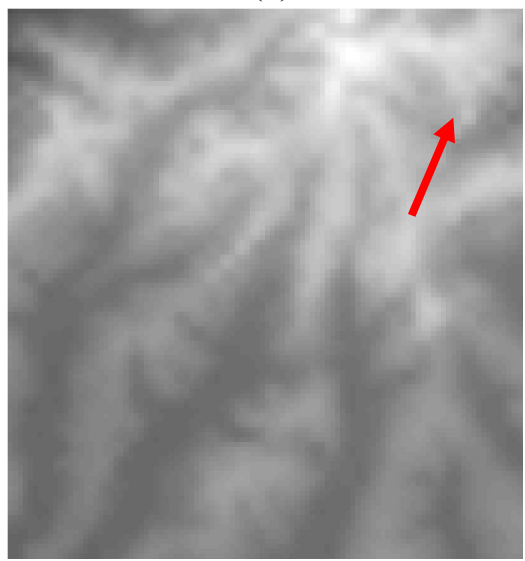

(e)

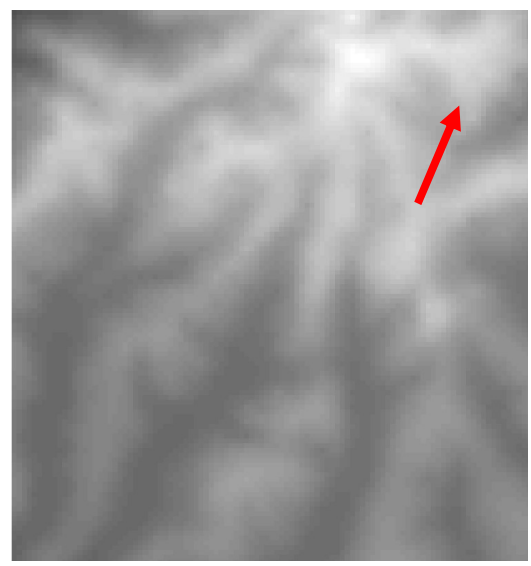

(h)

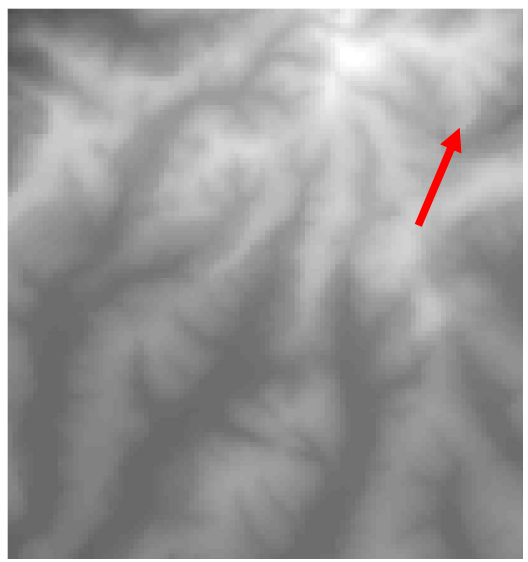

(c)

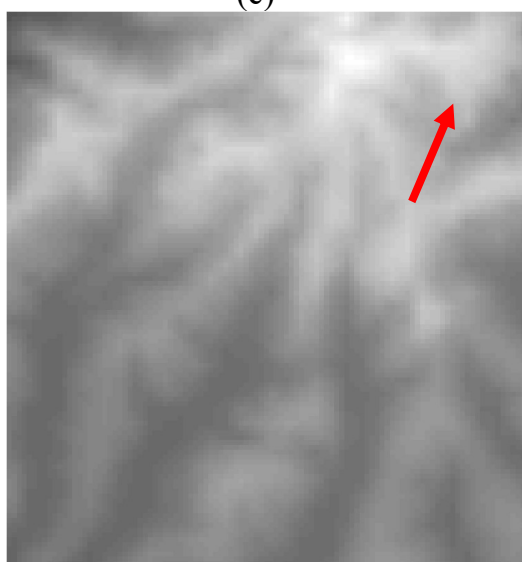

(f)

Fig. 6 Downscaling of DEM data from $90 \mathrm{~m}$ to $30 \mathrm{~m}$ spatial resolution (S2 dataset). (a) Contour data at $5 \mathrm{~m}$ interval; (b) Contour data at $10 \mathrm{~m}$ interval; (c) Reference DEM data at $20 \mathrm{~m}$ resolution (created from $5 \mathrm{~m}$ internal contour data); (d) Input DEM data at $90 \mathrm{~m}$ resolution (created from $10 \mathrm{~m}$ internal contour data) (note: this forms the only input to the algorithms); (e) HNN downscaled DEM at $30 \mathrm{~m}$ resolution; (f) DEM at $30 \mathrm{~m}$ resolution resulted from bilinear resampling; (g) DEM at $30 \mathrm{~m}$ resolution resulted from bi-cubic resampling; and (h) DEM at $30 \mathrm{~m}$ resolution resulted from Kriging resampling.

newly proposed HNN method are visually more similar to the reference DEMs than the input coarse spatial resolution DEMs and the DEMs generated by the bilinear, bi-cubic and Kriging benchmark methods for both the degraded and the sampled datasets. The improvement in visual similarity between the downscaled DEMs and reference DEM is seen clearly when comparing the $20 \mathrm{~m}$ DEM of the D1 dataset (Fig. 3), $30 \mathrm{~m}$ DEM of the D2 dataset (Fig. 4), $5 \mathrm{~m}$ DEM of the S1 dataset (Fig. 5) and $30 \mathrm{~m}$ DEM of the S2 dataset (Fig. 6) with the respective reference DEMs. The images of the input coarse resolution DEMs and the DEMs produced by the benchmark methods, especially the images created by bi-cubic resampling, are blurred with noise and the shapes of terrain features in these images look distorted. 


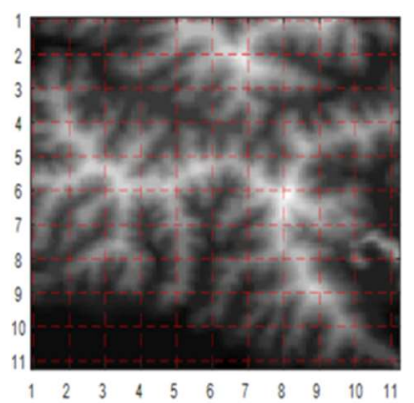

(a)

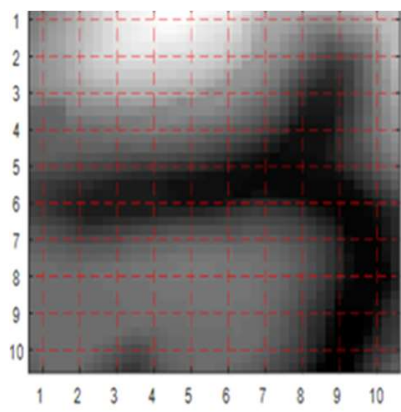

(c)

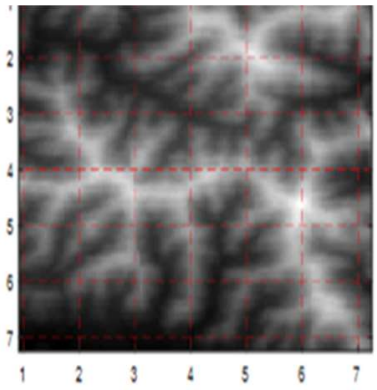

(b)

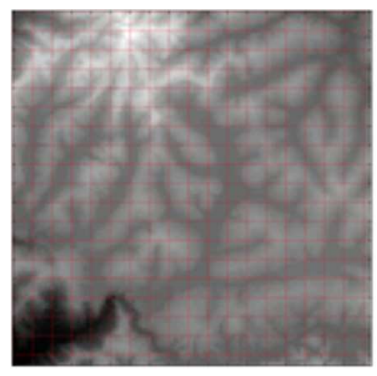

(d)
Fig. 7 The positions of profiles for DEM accuracy evaluation: (a) D1 dataset; (b) D2 dataset; (c) S1 dataset; and (d) S2 dataset.

In contrast, the images of the HNN downscaled DEMs in Fig. 3(c), Fig. 4(c), Fig. 5(c) and Fig. 6(e) look free of noise and similar to the reference DEMs in Fig. 3(a), Fig. 4(a), Fig. 5(a), and Fig. 6(c). The most clear improvement in the reconstruction of the shape of the terrain from the input coarse resolution data can be seen in the marked areas in Fig. 3, Fig. 4, Fig. 5, and Fig. 6. Comparison of the surfaces of the resulting DEMs using profiles from the locations shown in Fig. 7 reveals the clear advantage of the HNN downscaling method over the benchmark resampling methods. The elevation profiles of the HNN downscaled DEMs are closer to the profiles of the reference DEMs than those of the benchmark DEMs for all four datasets. This is most clearly seen in the D1 dataset in (a) (an example column profile) and (b) (an example row profile) in places such as tops of hills or bottoms of valleys and S1 dataset (c) (an example column profile) and (d) (an example row profile) (Fig. 9). In these images, it is possible to see that the surfaces formed by the resampling methods are closer to the input coarse spatial resolution surface while the surface formed by the HNN downscaled DEM is closer to the $5 \mathrm{~m}$ reference surface. The HNN downscaling method performed much more accurately than the benchmark resampling methods for more extreme elevation features such as the tops of ridges and hills or bottoms of valleys, especially for V-shaped valleys and sharp ridges and hills. This can be explained by the effects of the elevation constraint which helps to reduce or increase the elevation at such points while the spatial dependence maximization function ensures that the elevations of the adjacent sub-pixels change gradually, as in the real terrain.

Visual comparison of the scatterplots in Fig. 9 and Fig. 10 also show the closer match between the results of the HNN downscaling method and the reference DEM data in comparison with the input coarse DEM and benchmark results.
TABLE 2

ROOT MEAN SQUARED ERROR FOR BILINEAR, BI-CUBIC, KRIGING RESAMPLING AND HNN DOWNSCALING

\begin{tabular}{|c|c|c|c|c|c|c|}
\hline Da & asets & $\begin{array}{c}\text { Bilinear } \\
(\mathrm{m})\end{array}$ & $\begin{array}{l}\text { Bi-cubic } \\
\text { (m) }\end{array}$ & $\begin{array}{c}\text { Kriging } \\
(\mathrm{m})\end{array}$ & $\begin{array}{c}\mathrm{HNN} \\
(\mathrm{m})\end{array}$ & $\begin{array}{c}\text { Accuracy } \\
\text { improve- } \\
\text { ment over } \\
\text { Bilinear } \\
(\%)\end{array}$ \\
\hline & $\begin{array}{l}\text { Overall } \\
\text { RMSE }\end{array}$ & 3.3026 & 3.3716 & 2.8874 & 1.9853 & $39.9 \%$ \\
\hline vghe An & Min CP & 2.5245 & 2.5619 & 2.6330 & 1.9124 & $24.2 \%$ \\
\hline & Max CP & 2.9851 & 3.0731 & 2.7065 & 2.0171 & $32.4 \%$ \\
\hline & Min RP & 2.8843 & 2.9332 & 2.8899 & 1.9631 & $31.9 \%$ \\
\hline & Max RP & 2.9903 & 3.0293 & 2.8799 & 1.7544 & $41.3 \%$ \\
\hline & $\begin{array}{l}\text { Overall } \\
\text { RMSE }\end{array}$ & 8.8105 & 8.8736 & 8.5719 & 8.3510 & $5.2 \%$ \\
\hline $\begin{array}{l}30 \mathrm{~m} \\
\text { Sohe A }\end{array}$ & Min CP & 10.7635 & 10.8141 & 11.0702 & 10.4270 & $3.1 \%$ \\
\hline Nghe A & Max CP & 6.8408 & 6.9101 & 6.9668 & 6.4673 & $5.5 \%$ \\
\hline & Min RP & 6.4032 & 6.4005 & 6.2829 & 6.3202 & $1.3 \%$ \\
\hline & Max RP & 8.0953 & 8.0897 & 8.0478 & 7.6225 & $5.8 \%$ \\
\hline & $\begin{array}{l}\text { Overall } \\
\text { RMSE }\end{array}$ & 1.5139 & 1.6000 & 1.2092 & 0.8493 & $43.9 \%$ \\
\hline $5 \mathrm{~m}$ & Min CP & 1.2419 & 1.2912 & 0.8727 & 0.9734 & $21.6 \%$ \\
\hline $\begin{array}{l}\text { ang Son } \\
\text { (S1) }\end{array}$ & Max CP & 1.1635 & 21 & 1.1771 & 0.5120 & $56.0 \%$ \\
\hline & Min RP & 1.4081 & 1.4297 & 1.4138 & 1.1131 & $21.0 \%$ \\
\hline & Max RP & 1.4361 & 1.5174 & 1.6807 & 0.5897 & $58.9 \%$ \\
\hline & $\begin{array}{l}\text { Overall } \\
\text { RMSE }\end{array}$ & 84 & 18 & 95 & 46 & $10.0 \%$ \\
\hline $\begin{array}{l}30 \mathrm{~m} \\
\text { Dac La }\end{array}$ & Min CP & 2.2395 & 2.2698 & 2.1836 & 2.3247 & $-3.8 \%$ \\
\hline (S2) & Max CP & 1377 & .2514 & 1.0253 & 0.9323 & $18.1 \%$ \\
\hline & Min RP & 1.5355 & 1.5676 & 1.3829 & 1.6376 & $-6.6 \%$ \\
\hline & Max RP & 1.2816 & 1.4015 & 1.1203 & 1.3186 & $-2.9 \%$ \\
\hline
\end{tabular}

*Overall RMSE stands for Overall Root Mean Square Error

* Min CP stands for the Column Profile with Min Accuracy Improvement

* Max CP stands for the Column Profile with Max Accuracy Improvement

* Min RP stands for the Row Profile with Min Accuracy Improvement

* Max RP stands for the Row Profile with Max Accuracy Improvement

In these scatterplots, the two DEM datasets are considered to be closer if the data points are located closer to the regression line. Additionally, the slope coefficient $m$ should be closer to the value of 1 and the intercept coefficient $b$ closer to the value of 0 . The scatterplots of the HNN downscaling results in Fig. 9(a) and Fig. 10(a) show a closer match between the reference DEM and the HNN downscaled DEM data in comparison with the bilinear (Fig. 9(b) and Fig. 10(b), bi-cubic resampling (Fig. 9(c) and Fig. 10(c)), Kriging resampling (Fig. 9(d) and Fig. 10(d)) DEM data. This increase in accuracy can be seen most clearly with the $5 \mathrm{~m} \mathrm{~S} 1$ dataset and $20 \mathrm{~m}$ D1 DEM which produced a closeness to the best fit line and the regression coefficients ( $m$, $b$ ) in these scatterplots are closer to the values of 1 and 0 . Comparing the two datasets, the data points in the scatterplots in Fig. 9(c) and Fig. 10(c) (bilinear resampled DEM), Fig. 9(d) and Fig. 10(d) (bi-cubic resampled DEM), Fig. 9(e) and Fig. 10(e) (Kriging resampled DEM) are more scattered away from the 1:1 line than those of the HNN downscaled DEMs.

\section{3) Quantitative assessment}

Coinciding with the result of visual observation, quantitative assessment based on the RMSE (Table 2) reveals the greater accuracy of the HNN downscaling method over the benchmark methods for all four datasets. Among the two degraded datasets (D1 and D2 datasets), the increase in accuracy is greater for the D1 dataset. The RMSE for the HNN downscaling DEM is $1.9853 \mathrm{~m}$ while the RMSEs for the bilinear, bi-cubic and Kriging resampling methods are 3.3716 m, $3.3716 \mathrm{~m}$ and 


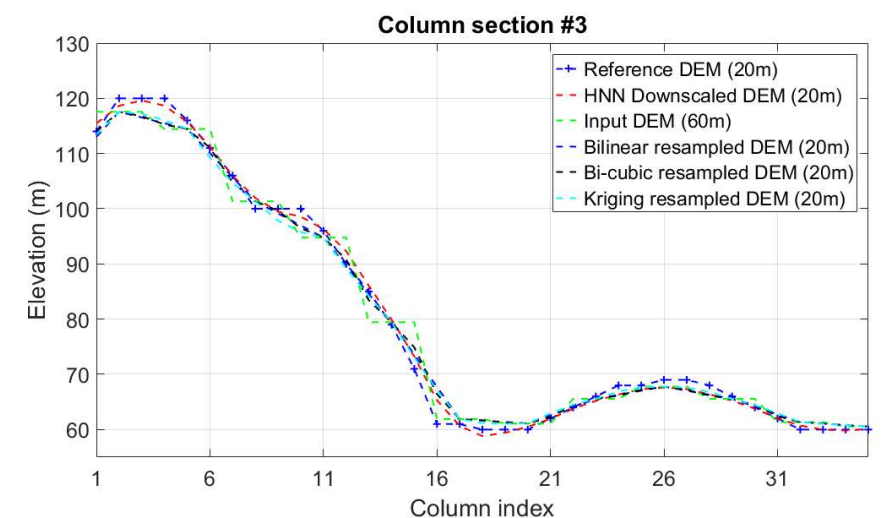

(a)

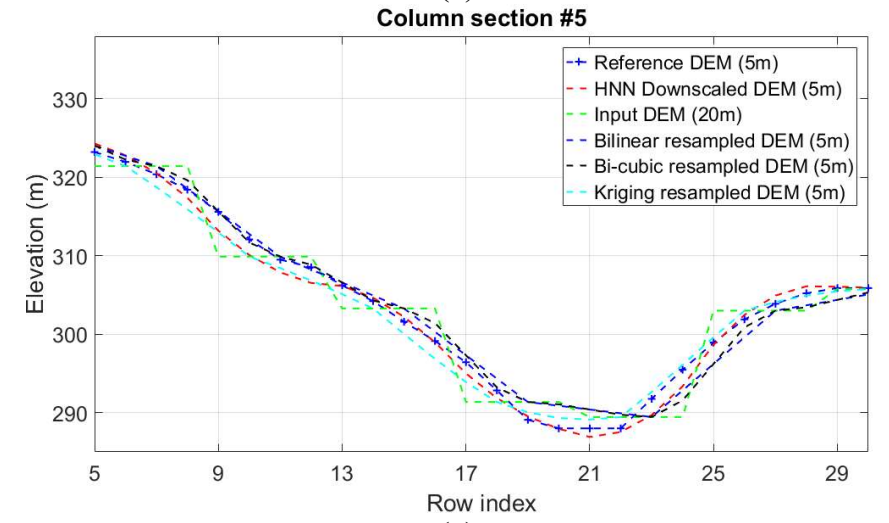

(c)

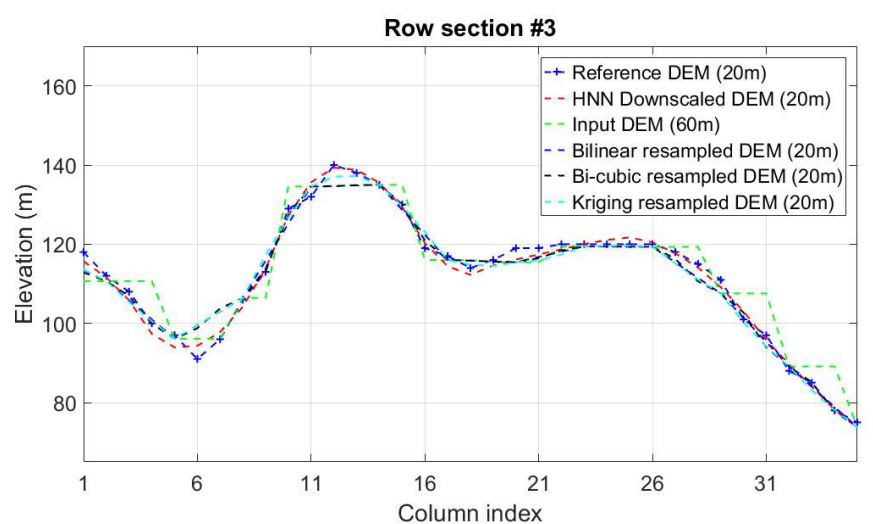

(b)

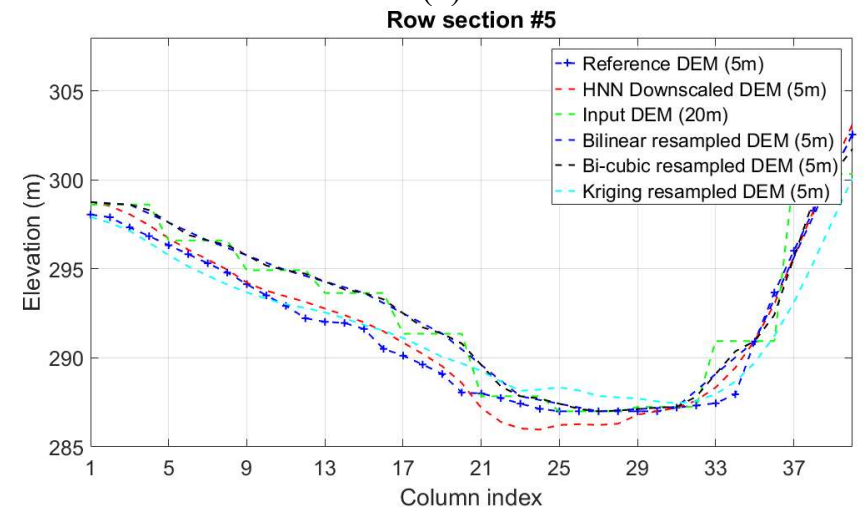

(d)

Fig. 8 Comparison of reference surface (reference DEM), HNN downscaled surface (downscaled DEM), input coarse resolution surface (input-DEM), bilinear, bicubic and Kriging resampled surfaces based on profiles: (a) a column profile for the D1 dataset; (b) a row profile for the D1 dataset, (e) a column profile for the S1 dataset; (f) a row profile for the S1 dataset.

$2.8874 \mathrm{~m}$, respectively. Comparing with the RMSE of the 20 $\mathrm{m}$ DEM obtained by bilinear resampling from the $60 \mathrm{~m}$ data of Nghe An (D1 dataset), the RMSE of the HNN downscaled 20 m reduced significantly by $39.9 \%$. For the $30 \mathrm{~m}$ D2 dataset test, the increase in accuracy for the HNN downscaling algorithm is not as large as for the other datasets but is still convincing with the RMSE reduced by 5\% compared with the $30 \mathrm{~m}$ DEM obtained by bilinear resampling from the input $90 \mathrm{~m}$ DEM. The increase in accuracy for the sampled DEMs is similar to that for the degraded DEMs. The RMSE of the $5 \mathrm{~m}$ Lang Son data (S1 dataset) decreased sharply for the HNN downscaling DEM to $0.8493 \mathrm{~m}$ from $1.5139 \mathrm{~m}$ for the $5 \mathrm{~m}$ DEM obtained by bilinear resampling (43.9\%), $1.6 \mathrm{~m}$ for bi-cubic resampling, and 1.2092 $\mathrm{m}$ for Kriging resampling. The result for the $\mathrm{S} 2$ test dataset is not as impressive as that for the S1 dataset. However, the increase in DEM accuracy is significant with the RMSE reduced by $5.2 \%$ in comparison with the bilinear resampled DEM. These statistics show that the proposed HNN method can increase the sub-pixel accuracy of a gridded DEM by downscaling. Furthermore, the HNN model in the presented examples appeared to work more effectively with the finer spatial resolution DEMs.

The increase in accuracy in terms of the RMSE, along with the profiles, showed the effects of terrain features on the performance of the HNN algorithm. For the $20 \mathrm{~m}$ D1 dataset and $30 \mathrm{~m} \mathrm{D} 2$ dataset in Nghe An, the increase in accuracy between the resampled and downscaled DEMs is relatively constant. For the $30 \mathrm{~m}$ sampled dataset (S2 dataset), the increase in accuracy for most profiles is between $10 \%$ and for the $5 \mathrm{~m}$ sampled Lang Son dataset (S1 dataset) is more variable with a smallest value of $21 \%$ and largest value of $58 \%$. This is because most of the profiles with a large increase in accuracy of more than $50 \%$ (such as column cross-sections 2, 4 and row cross-sections 2, 4, 9 (Fig. 7)) are located in areas of specific terrain such as valley bottoms or the tops of hills. In contrast, the profiles with a smaller increase in accuracy occur mostly on the sides of mountains where the surface represented by the input DEM is relatively close to the reference (fine) DEM. The smaller variation in the accuracy increase for the $20 \mathrm{~m}$ and 30 $\mathrm{m}$ degraded (D1 and D2) DEMs and $30 \mathrm{~m} \mathrm{~S} 2$ DEMs occurs because most profiles are located in different types of terrain rather than occurring mostly in specific terrain forms. The range of the increase in accuracy (comparing with bilinear) for the 20 $\mathrm{m}$ dataset is $16 \%$ and between $24 \%$ and $41 \%$.

The similarity of the two DEMs can also be evaluated quantitatively using the linear regression coefficients $(m, b)$ and the correlation coefficient $R$. Because the value of $m$ may be greater or smaller than 1 and the value of $b$ may be greater or smaller than 0, comparison between different values of $m$ and $b$ was undertaken using the sub-parameters $|1-m|$ and $|b|$. Accordingly, smaller values of $|1-m|$ and $|b|$ simultaneously indicate greater similarity between two datasets. The third parameter for evaluating the fit of the two datasets is the correlation coefficient $R$. The correlation coefficient measures 


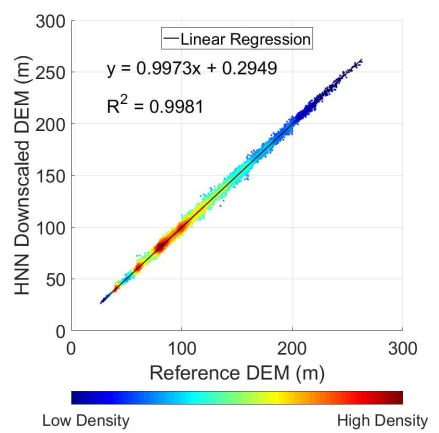

(a)

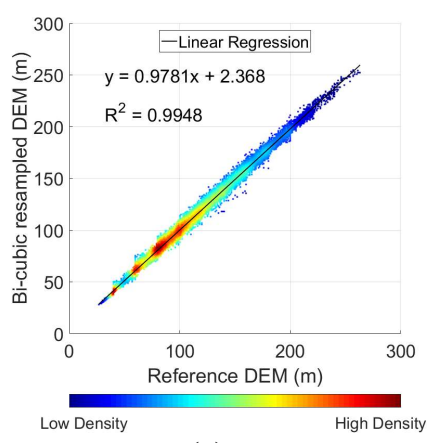

(c)

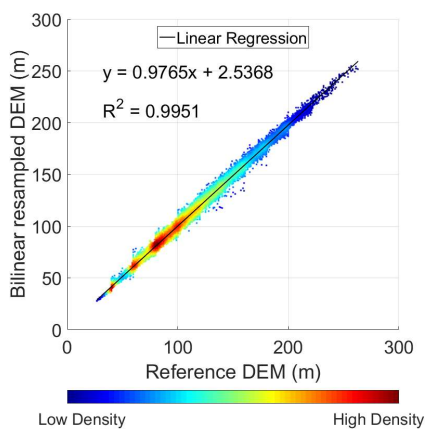

(b)

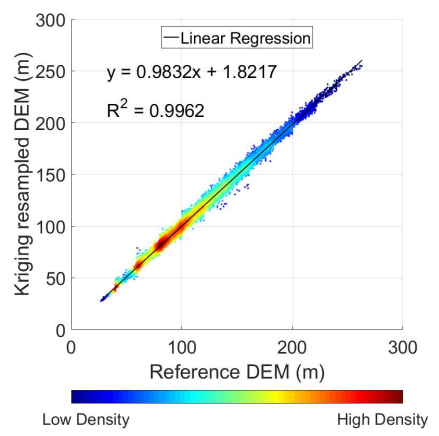

(d)

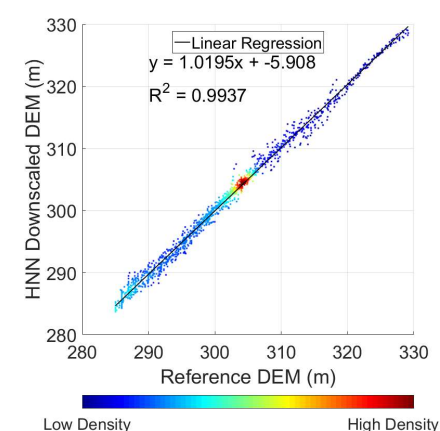

(a)

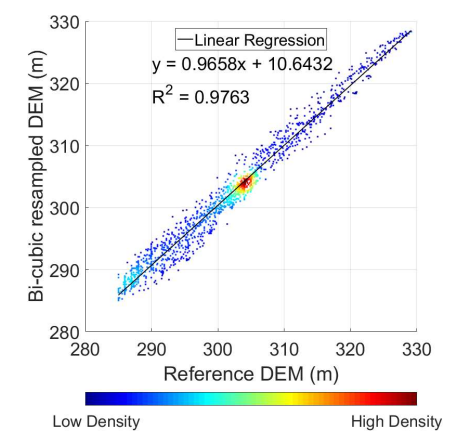

(c)

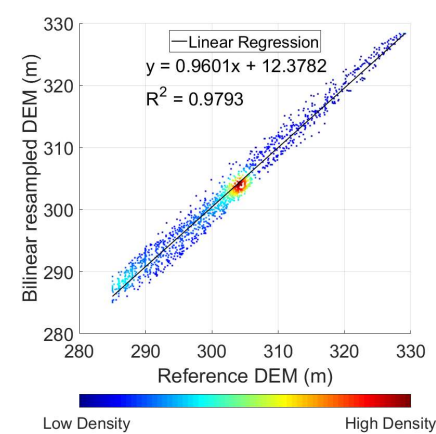

(b)

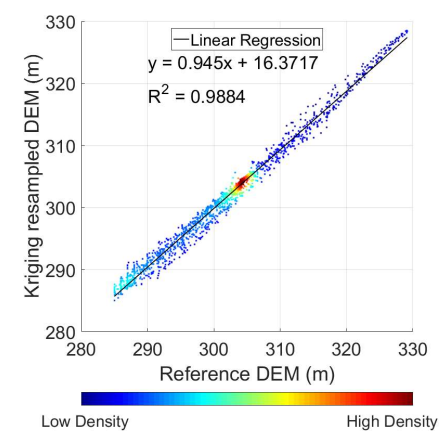

(d)

Fig. 10 Scatterplots of the reference fine spatial resolution DEM against the downscaled DEMs for the S1 dataset: (a) HNN downscaled DEM; bilinear resampled DEM; (c) bi-cubic resampled DEM; (d) Kriging resampled DEM.

the association between two datasets and, thus, captures the distribution of the data points in the scatterplots around the best fit line. The closer the value of $R^{2}$ to 1 , the more data points are located close to the best fit line. A perfect match between two DEM datasets means that all the data points are located on the identity line $(y=x)$ and the coefficient of determination $R^{2}=1$. That means the two datasets are exactly the same if the value of $m$ is equal to $1, b$ is equal to 0 and $R^{2}$ is equal to 1 , simultaneously.

To evaluate the results of the different methods, linear regression models were fitted to the relations between the reference data and the downscaled datasets (Table 3). The coefficient values show the better fitting of the HNN downscaled DEMs with the reference DEMs than those of the benchmark resampled DEMs. For all four datasets, the values of the parameters $m, b$ and $R^{2}$ of the HNN downscaled DEMs are much closer to the values of 1,0 , and 1 , respectively, than those of the bilinear, bi-cubic, and Kriging resampled DEMs. For the Lang Son (S1 dataset) DEMs, the values $|1-m|=$ $0.0195,|b|=5.9080$ and $R^{2}=0.9937$ for the HNN downscaled DEM showed greater similarity to the reference DEM than those of the bilinear resampled DEM $(|1-m|=0.0399,|b|=$ 12.3782 and $\left.R^{2}=0.9793\right)$, bi-cubic resampled DEM $(|1-m|$ $=0.0342,|b|=10.6432$ and $\left.R^{2}=0.9763\right)$, and Kriging resampled DEM $\left(|1-m|=0.0550,|b|=16.3717\right.$ and $R^{2}=$ 0.9884). Linear regression statistics for the $\mathrm{Dac} \mathrm{Ha}$ (S2 dataset) sampled data also showed the better match of the downscaled DEM to the reference DEM with $|1-m|=0.0043,|b|=$ 4.1179 and $R^{2}=0.9968$ compared with $|1-m|=0.0128,|b|=$ 12.1453 and $R^{2}=0.9960$ for bilinear resampling, $|1-m|=$ $0.0115,|b|=10.9118$ and $R^{2}=0.9959$ for bi-cubic resampling,

Fig. 9 Scatterplots of the reference fine spatial resolution DEM against the downscaled DEMs for the D1 dataset: (a) HNN downscaled DEM; (b) bilinear resampled DEM; (c) bi-cubic resampled DEM; (d) Kriging resampled DEM.

and $|1-m|=0.0078,|b|=7.3917$ and $R^{2}=0.9967$ for Kriging resampling.

Linear regression coefficients for the $20 \mathrm{~m}$ Nghe An degraded (D1) dataset showed that the HNN downscaled DEM matches very closely to the reference DEM with $|1-m|=$ $0.0019,|b|=0.2949$ and $R^{2}=0.9973$ whereas the other downscaled DEMs are very different to the reference DEM.

For the $30 \mathrm{~m}$ degraded (D2) dataset, the increase in prediction precision of the HNN downscaling is seen clearly when comparing the linear regression parameters of the four other methods. Although the coefficient of determination of the HNN downscaling result $\left(R^{2}=0.9686\right)$ is just slightly larger than those of the bilinear $\left(R^{2}=0.9646\right)$ and bi-cubic $\left(R^{2}=\right.$ $0.9639)$ methods and even smaller than that of Kriging $\left(R^{2}=\right.$ 0.9694), the best fit lines of the datasets showed less bias for the HNN downscaled DEM data with the reference data $(|1-m|$ $=0.0096$ and $|b|=1.6013)$ than those of the bilinear resampling $(|1-m|=0.0500$ and $|b|=3.2057)$, bi-cubic resampling $(|1-m|=0.0471$ and $|b|=2.8723)$, and Kriging interpolation $(|1-m|=0.0392$ and $|b|=1.9291)$ data.

Comparing the slope parameter $m$ and intercept parameter $b$ of the best fit lines of all four datasets, it is clear that all the slope parameters $m$ of the resampled DEMs are smaller than 1 and the intercept parameters $b$ are larger than 0 . This means that for locally-low places (usually the bottom of valleys) the pixels of the DEM data produced by these methods are likely to be higher than the corresponding pixels in the reference DEM. Conversely, for locally-high places such as the tops of hills or mountain ridges, the elevations of the pixels in the resampled DEM data are likely to be lower than that of the corresponding pixels in the reference image. This is due to the smoothing 
TABLE 3

LINEAR REGRESSION COEFFICIENTS FOR ALL FOUR DATASETS

\begin{tabular}{llccc}
\hline \multirow{2}{*}{ Datasets } & \multicolumn{3}{c}{ Linear Regression Coefficients } \\
\cline { 2 - 5 } & & $m$ & $b$ & $\mathrm{R}^{2}$ \\
\hline \multirow{3}{*}{$\begin{array}{l}\text { 20 m Nghe An } \\
\text { (D1) dataset }\end{array}$} & $20 \mathrm{~m}$ downscaled & 0.9973 & 0.2949 & 0.9981 \\
\cline { 2 - 5 } & $20 \mathrm{~m}$ bilinear & 0.9765 & 2.5368 & 0.9951 \\
\cline { 2 - 5 } & $20 \mathrm{~m}$ bi-cubic & 0.9781 & 2.3680 & 0.9948 \\
\cline { 2 - 5 } & $20 \mathrm{~m}$ Kriging & 0.9832 & 1.8217 & 0.9962 \\
\hline \multirow{3}{*}{$\begin{array}{l}\text { 30 m Nghe An } \\
\text { (D2) dataset }\end{array}$} & $30 \mathrm{~m}$ downscaled & 0.9904 & -1.6013 & 0.9686 \\
\cline { 2 - 5 } & $30 \mathrm{~m}$ bilinear & 0.9500 & 3.2057 & 0.9646 \\
\cline { 2 - 5 } & $30 \mathrm{~m}$ bi-cubic & 0.9529 & 2.8723 & 0.9639 \\
\hline \multirow{3}{*}{$\begin{array}{l}\text { Lang Son (S1) } \\
\text { dataset }\end{array}$} & $30 \mathrm{~m}$ Kriging & 0.9608 & 1.9291 & 0.9694 \\
\cline { 2 - 5 } & $5 \mathrm{~m}$ downscaled & 1.0195 & -5.908 & 0.9937 \\
\hline & $5 \mathrm{~m}$ bilinear & 0.9601 & 12.3782 & 0.9793 \\
\hline \multirow{3}{*}{$\begin{array}{l}\text { Dac Ha (S2) } \\
\text { dataset }\end{array}$} & $5 \mathrm{~m}$ Kriging & 0.9658 & 10.6432 & 0.9763 \\
\cline { 2 - 5 } & $30 \mathrm{~m}$ downscaled & 1.0043 & -4.1179 & 0.9968 \\
\cline { 2 - 5 } & $30 \mathrm{~m}$ bilinear & 0.9872 & 12.1453 & 0.9960 \\
\hline & $30 \mathrm{~m}$ bi-cubic & 0.9885 & 10.9118 & 0.9959 \\
\hline & $30 \mathrm{~m}$ Kriging & 0.9922 & 7.3917 & 0.9967 \\
\hline
\end{tabular}

effect (referred to as conditional bias where highs are underpredicted and lows are over-predicted) and can be reduced using $\mathrm{HNN}$ downscaling. The evidence for this is in the values of the four pairs of $m$ and $b$ values for the HNN downscaling method for the $20 \mathrm{~m}(m=0.9981, b=0.2949)$ and $30 \mathrm{~m}(m=$ $0.9904, b=-1.6013)$ degraded DEMs, and $5 \mathrm{~m}(m=1.0195, b$ $=-5.9080)$ and $30 \mathrm{~m}(m=1.0043, b=-4.1179)$ sampled DEMs. These best fit lines are very close to the 1:1 line with $m=1$, and $b=0$. Even for the $5 \mathrm{~m} \mathrm{D} 1$ dataset, the HNN downscaling method has a tendency to produce elevation values in low elevation areas that are slightly lower and elevation values in high elevation areas that are slightly higher than those of the reference DEM. This can be explained by the effect of the elevation constraint of the HNN downscaling model. This effect is crucial, as it reveals that the HNN approach works not because it is an alternative spatial smoother that captures more of the salient information in the coarse resolution data, but explicitly because it imposes a pixel-level constraint on the predictions such that extremes tend to be more closely honoured. In other words, the structure of the HNN method (formulated as a within- and across-pixel smoothing goal and pixel-level constraint) means that it brings a specific advantage that other commonly applied resampling and interpolation methods do not.

Although the proposed HNN model for downscaling DEMs has been demonstrated to be more accurate than conventional resampling methods, the algorithm has some limitations. In particular, because the HNN model uses a linear activation function there is no tolerance in the constraint as in the HNN model for sub-pixel mapping. Thus, if the input coarse DEM contains a large error, this error may be fully transferred to the downscaled DEM. To solve this problem, further research and modification of the HNN model, especially the activation functions, is needed.

\section{CONCLUSION}

A new method for increasing the spatial pixel size and accuracy of gridded DEMs was proposed and tested comprehensively using data with different DEM spatial resolutions and characteristics. The newly proposed downscaling algorithm was formulated based on the Hopfield neural network (HNN) with a spatial dependence maximization goal function and an elevation constraint. Tests of the proposed HNN model were implemented on two types of elevation datasets; $20 \mathrm{~m}$ (D1) and $30 \mathrm{~m}$ (D2) degraded DEMs in Nghe An province, Vietnam, a $5 \mathrm{~m}$ sampled DEM (S1) in Lang Son province (from ground surveying elevation data), and $30 \mathrm{~m}$ sampled DEM (S2) in Dac Ha, Kontum Province, Vietnam (generated from contour lines). The proposed method was evaluated against three existing and commonly applied benchmark methods: the bilinear, bi-cubic and Kriging resampling methods.

The test results showed a sharp increase in accuracy for the HNN downscaled gridded DEMs in comparison with the bilinear, bi-cubic, and Kriging resampled DEMs. Visual assessment revealed the greater similarity of the HNN downscaled DEMs with the reference DEM than the DEMs generated by bilinear, bi-cubic and Kriging resampling. Quantitative accuracy assessment based on the RMSE showed an increase in DEM accuracy for the HNN downscaling algorithm over the bilinear, bi-cubic and Kriging resampling methods. The RMSE of the downscaled DEMs decreased by approximately $39.9 \%, 5.2 \%, 43.9 \%$, and $10.0 \%$ for the $20 \mathrm{~m}$ and $30 \mathrm{~m}$ degraded DEMs in Nghe An province (D1 and D2 datasets), $5 \mathrm{~m}$ sampled DEM in Lang son province (S1 dataset), and $30 \mathrm{~m}$ sampled DEM in Dac Ha, Vietnam (S2 dataset), respectively. The overall RMSE values of the HNN downscaled DEM were smaller in comparison with those of the bilinear, bicubic and Kriging resampling methods, especially for the $5 \mathrm{~m}$ and $20 \mathrm{~m}$ datasets.

Further evaluation was also implemented using linear regression of the reference spatial resolution DEM against the HNN downscaled DEM and the benchmark resampled DEMs, particularly focusing on the coefficients $m, b$ and $R^{2}$. Analysis of these parameters showed that the HNN downscaled DEMs were closer to the reference DEMs than those produced using the benchmark methods.

Visual and quantitative assessment showed that the HNN downscaling algorithm performed more accurately for some specific terrain features such as valley bottoms or the crests of ridges. The RMSEs of profiles located mostly in these terrain features decreased by about $20 \%$ (i.e., improved more) compared with those of the profiles occurring mostly on mountain sides or flat areas. This improvement can be attributed to the effects of the combination of the elevation constraint with the spatial dependence maximization functions in the HNN approach. That is, the specific formulation of the HNN method brings structural advantages to the DEM downscaling task that cannot be achieved using commonly applied spatial resampling and interpolation methods.

REFERENCES 
[1] S. Saksena and V. Merwade, "Incorporating the effect of DEM resolution and accuracy for improved flood inundation mapping," J. Hydrol., vol. 530, pp. 180194, 2015.

[2] A. S. Reddy and M. J. Reddy, "Evaluating the influence of spatial resolutions of DEM on watershed runoff and sediment yield using SWAT," J. Earth Syst. Sci., vol. 124, no. 7, pp. 1517-1529, 2015.

[3] L. Bian and R. Butler, "Comparing Effects of Aggregation Methods on Statistical and Spatial Properties of Simulated Spatial Data," Photogramm. Eng. Remote Sens., vol. 65, no. 1, pp. 73-84, 1999.

[4] Z. Zhao, G. Benoy, T. L. Chow, H. W. Rees, J. L. Daigle, and F. R. Meng, "Impacts of accuracy and resolution of conventional and LiDAR based DEMs on parameters used in hydrologic modeling," Water Resour. Manag., vol. 24, no. 7, pp. 1363-1380, 2010.

[5] K. Chang and B. Tsai, "The Effect of DEM Resolution on Slope and Aspect Mapping," Cartogr. Geogr. Inf. Syst., vol. 18, no. 1, pp. 69-77, 2008.

[6] T. Bolstad, Paul V. \& Stowe, "An Evaluation Accuracy: of DEM Elevation, Slope , and Aspect," ISPRS J. Photogrametric Eng. Remote Sens., vol. 60, pp. 1327-1332, 1994.

[7] I. Chaubey, A. S. Cotter, T. A. Costello, and T. S. Soerens, "Effect of DEM data resolution on SWAT output uncertainty," Hydrol. Process., vol. 19, no. 3, pp. 621-628, 2005.

[8] K. S. Rawat, G. Krishna, A. Mishra, J. Singh, and S. V. Mishra, "Effect of DEM data resolution on low relief region sub-watershed boundaries delineating using of SWAT model and DEM derived from CARTOSAT-1 (IRS-P5), SRTM and ASTER," J. Appl. Nat. Sci., vol. 6, no. 1, 2018.

[9] B. E. Vieux, "DEM Aggregation and Smoothing Effects on Surface Runoff Modeling," J. Comput. Civ. Eng., vol. 7, no. 3, 2006.

[10] R. F. Vázquez and J. Feyen, "Assessment of the effects of DEM gridding on the predictions of basin runoff using MIKE SHE and a modelling resolution of 600 m," J. Hydrol., vol. 334, no. 1-2, pp. 73-87, 2007.

[11] E. Ray, Learning XML. Beijing; Cambridge, Mass: O'Reilly, 2001.

[12] J. Li and D. W. S. Wong, "Effects of DEM sources on hydrologic applications," Comput. Environ. Urban Syst., vol. 34, no. 3, pp. 251-261, 2010.

[13] M. Sulis, C. Paniconi, and M. Camporese, "Impact of grid resolution on the integrated and distributed response of a coupled surface-subsurface hydrological model for the des Anglais catchment, Quebec," Hydrol. Process., vol. 25, no. 12, pp. 1853-1865, 2011.

[14] M. P. Smith, A. X. Zhu, J. E. Burt, and C. Stiles, "The effects of DEM resolution and neighborhood size on digital soil survey," Geoderma, vol. 137, no. 1-2, pp. 58-69, 2006.

[15] W. L. Kuo et al., "Effect of grid size on runoff and soil moisture for a variable-source-area hydrology model," Water Resour. Res., vol. 35, no. 11, pp. 3419-3428, 1999.

[16] Q. Guo, W. Li, H. Yu, and O. Alvarez, "Effects of
Topographic Variability and Lidar Sampling Density on Several DEM Interpolation Methods," Photogramm. Eng. Remote Sens., vol. 76, no. 6, pp. 701-712, 2013.

[17] J. P. Wilson, "Digital terrain modeling," Geomorphology, vol. 137, no. 1, pp. 107-121, 2012.

[18] S. Rapinel, L. Hubert-Moy, B. Clément, J. Nabucet, and C. Cudennec, "Ditch network extraction and hydrogeomorphological characterization using LiDAR-derived DTM in wetlands," Hydrol. Res., 2015.

[19] X. Liu, "Airborne LiDAR for DEM generation: Some critical issues," Progress in Physical Geography. 2008.

[20] D. Kidner, M. Dorey, and D. Smith, "What's the point? Interpolation and extrapolation with a regular grid DEM," in GeoComputation.org, 1999.

[21] S. Wu, J. Li, and G. H. Huang, "A study on DEMderived primary topographic attributes for hydrologic applications: Sensitivity to elevation data resolution," Appl. Geogr., 2008.

[22] W. Shi, B. Wang, and Y. Tian, "Accuracy Analysis of Digital Elevation Model Relating to Spatial Resolution and Terrain Slope by Bilinear Interpolation," Math. Geosci., vol. 46, no. 4, pp. 445-481, 2014.

[23] C. H. Grohmann and S. S. Steiner, "SRTM resample with short distance-low nugget kriging," Int. J. Geogr. Inf. Sci., vol. 22, no. 8, pp. 895-906, 2008.

[24] B. Dixon and J. Earls, "Resample or not?! Effects of resolution of DEMs in watershed modeling," Hydrol. Process., 2009.

[25] L. E. Band and I. D. Moore, "Scale: Landscape attributes and geographical information systems," Hydrol. Process., vol. 9, no. 3-4, pp. 401-422, 1995.

[26] W. G. Rees, "The accuracy of Digital Elevation Models interpolated to higher resolutions," Int. J. Remote Sens., vol. 21, no. 1, pp. 7-20, 2000.

[27] R. Jana, T. V Reshmidevi, P. S. Arun, and T. I. Eldho, "An enhanced technique in construction of the discrete drainage network from low-resolution spatial database," Comput. Geosci., vol. 33, no. 6, pp. 717727, 2007.

[28] G. Jordan, "Adaptive smoothing of valleys in DEMs using TIN interpolation from ridgeline elevations: An application to morphotectonic aspect analysis," Comput. Geosci., vol. 33, no. 4, pp. 573-585, 2007.

[29] C. U. Paredes-Hernández, N. J. Tate, K. J. Tansey, P. F. Fisher, and W. E. Salinas-Castillo, "Increasing the Accuracy of Low Spatial Resolution Digital Elevation Models using Geostatistical Conflation," in Ninth International Symposium on Spatial Accuracy Assessment in Natural Resources and Environmental Sciences, 2010, pp. 413-416.

[30] Y. Tang, J. Zhang, H. Li, H. Ding, J. Liu, and L. Jing, A multiple-point geostatistical method for digital elevation models conflation. 2014.

[31] I. B. Atkins, M. View, T. Morgan, and W. Frank, "United States Patent (19)," no. 19, 2000.

[32] P. Atkinson, "Mapping sub-pixel boundaries from remotely sensed images," in Innovations in GIS 4, 4th ed., vol. 69, no. 4, Z. Kemp, Ed. London, Bristol,: Taylor \& Francis, 1997, pp. 152-169.

[33] Y. Su, G. M. Foody, A. M. Muad, and K. Cheng, 
"Combining Hopfield Neural Network and Contouring Methods to Enhance Super-Resolution Mapping," IEEE J. Sel. Top. Appl. Earth Obs. Remote Sens., vol. 5, no. 5, pp. 1403-1417, 2012.

[34] M. W. Thornton, P. M. Atkinson, and D. A. Holland, "Sub-pixel mapping of rural land cover objects from fine spatial resolution satellite sensor imagery using super-resolution pixel-swapping," Int. J. Remote Sens., vol. 27, no. 3, pp. 473-491, Feb. 2006.

[35] C. Huang, Y. Chen, and J. Wu, "DEM-based modification of pixel-swapping algorithm for enhancing floodplain inundation mapping," Int. J. Remote Sens., vol. 35, no. 1, pp. 365-381, Jan. 2014.

[36] L. Wang and Q. Wang, "Subpixel Mapping Using Markov Random Field With Multiple Spectral Constraints From Subpixel Shifted Remote Sensing Images," IEEE Geosci. Remote Sens. Lett., vol. 10, no. 3, pp. 598-602, 2013.

[37] A. J. Tatem, H. G. Lewis, P. M. Atkinson, and M. S. Nixon, "Multiple-class land-cover mapping at the subpixel scale using a Hopfield neural network," Int. J. Appl. Earth Obs. Geoinf., vol. 3, no. 2, pp. 184-190, 2001.

[38] Q. M. Nguyen, P. M. Atkinson, and H. G. Lewis, "Super-resolution mapping using Hopfield Neural Network with panchromatic image," in Asian Conference on Remote Sensing, 2005.

[39] A. M. Muad and G. M. Foody, "Super-resolution mapping using multiple observations and Hopfield neural network," in Proc.SPIE, 2010, vol. 7830.

[40] X. Li, F. Ling, Y. Du, Q. Feng, and Y. Zhang, "A spatial-temporal Hopfield neural network approach for super-resolution land cover mapping with multitemporal different resolution remotely sensed images," ISPRS J. Photogramm. Remote Sens., vol. 93, pp. 7687, 2014.

[41] Q. Wang, W. Shi, P. M. Atkinson, and Z. Li, "Land Cover Change Detection at Subpixel Resolution With a Hopfield Neural Network," IEEE J. Sel. Top. Appl. Earth Obs. Remote Sens., vol. 8, no. 3, pp. 1339-1352, 2015.

[42] Q. M. Nguyen, V. D. Do, P. M. Atkinson, and H. G. Lewis, "Downscaling Multispectral Imagery Based on the HNN Using Forward Model," no. October 2009, pp. 19-22.

[43] Q. M. Nguyen, P. M. Atkinson, and H. G. Lewis, "Super-resolution mapping using Hopfield Neural Network with panchromatic imagery," Int. J. Remote Sens., vol. 32, no. 21, pp. 6149-6176, Nov. 2011.

[44] D. W. Tank and J. J. Hopfield, "Simple 'Neural' Optimization Networks: an a/D Converter, Signal Decision Circuit, and a Linear Programming Circuit.," IEEE Trans. circuits Syst., vol. CAS-33, no. 5, pp. 533$541,1986$.

[45] S. Kienzle, "The Effect of DEM Raster Resolution on First Order, Second Order and Compound Terrain Derivatives," Trans. GIS, vol. 8, no. 1, pp. 83-111, 2003.

[46] U. Alganci, B. Besol, and E. Sertel, "Accuracy Assessment of Different Digital Surface Models,"
ISPRS Int. J. Geo-Information, vol. 7, no. 3, p. 114, 2018.

[47] P. M. Atkinson, E. Pardo-Iguzquiza, and M. ChicaOlmo, "Downscaling Cokriging for Super-Resolution Mapping of Continua in Remotely Sensed Images," IEEE Trans. Geosci. Remote Sens., vol. 46, no. 2, pp. 573-580, 2008.

[48] K. Whitehead and C. H. Hugenholtz, "Applying ASPRS Accuracy Standards to Surveys from Small Unmanned Aircraft Systems (UAS)," Photogramm. Eng. Remote Sens., vol. 81, no. 10, pp. 787-793, 2015.

[49] ASPRS, "ASPRS Positional Accuracy Standards for Digital Geospatial Data," Photogramm. Eng. Remote Sens., vol. 81, no. 3, 2015. 